\title{
聚酰亚胺基高比表面活性炭及其电化学性能研究
}

\author{
王 浩 ${ }^{1,2}$, 李 琳 $^{2}$, 王春雷 ${ }^{2}$, 王 倩 ${ }^{2}$, 梁长海 ${ }^{1,2}$, 王同华 ${ }^{2}$
}

(1. 大连理工大学 石油与化学工程学院, 盘锦 124221; 2. 大连理工大学 化工学院, 精细化工国家重点实验室, 炭 素材料研究室, 大连 116024)

摘 要: 以聚酰亚胺(PI)薄膜边角料为前驱体, 采用 $\mathrm{CO}_{2}$ 物理活化法制备高比表面活性炭。研究了活化工艺对 PI 活 性炭孔结构性能的影响及其活化机理，探讨了活性炭孔结构对其电化学性能的影响。结果表明, PI 薄膜可以在相对较 低的温度下经 $\mathrm{CO}_{2}$ 活化制备出具有无定型微晶质炭结构、孔隙结构发达的活性炭, 比表面积最高可达 $2809 \mathrm{~m}^{2} / \mathrm{g}$, 总 孔容积达 $1.423 \mathrm{~cm}^{3} / \mathrm{g}$; 通过控制 $\mathrm{CO}_{2}$ 活化工艺, 可实现对 PI 活性炭的孔道尺度与分布的调控。作为超级电容器电 极材料, PI 活性炭在 $100 \mathrm{~mA} / \mathrm{g}$ 条件下, 比电容高达 $237 \mathrm{~F} / \mathrm{g}$, 电容保持率为 $86 \%$ 。孔径集中于 $0.7 \sim 2 \mathrm{~nm}$, 并存在适 量介孔的活性炭具有极佳的电化学性能。

关 键 词: 聚酰亚胺薄膜; 活性炭; $\mathrm{CO}_{2}$ 活化; 高比表面积; 电化学性能

中图分类号: TQ127 文献标识码: A

\section{Preparation and Electrochemical Performance of Polyimide-based Activated Carbons with High Surface Area}

\author{
WANG Hao ${ }^{1}$, LI Lin ${ }^{2}$, WANG Chun-Lei ${ }^{2}$, WANG Qian ${ }^{2}$, LIANG Chang-Hai ${ }^{1,2}$, WANG Tong-Hua ${ }^{2}$ \\ (1. School of Petroleum and Chemical Engineering, Dalian University of Technology, Panjin 124221, China; 2. Carbon Re- \\ search Laboratory, State Key Laboratory of Fine Chemicals, School of Chemical Engineering, Dalian University of Technology, \\ Dalian 116024, China)
}

\begin{abstract}
Polyimide-based activated carbon (PIAC) was prepared by $\mathrm{CO}_{2}$ activation using polyimide (PI) film offcut as precursor. The influences of activation conditions on the pore structure of PI-based activated carbons and the activation mechanism were investigated. Effects of pore structure on electrochemical properties of PIAC were also investigated. Results show that the PI film offcuts can be used to prepare the PI-based activated carbon with high surface area, abundant porosity and amorphous carbon structure at relatively low temperature via $\mathrm{CO}_{2}$ activation, in which the highest specific surface area was $2809 \mathrm{~m}^{2} / \mathrm{g}$ with pore volume of $1.423 \mathrm{~cm}^{3} / \mathrm{g}$. Pore size and distribution of activated carbon can effectively be concentrated at $0.7-2 \mathrm{~nm}$ with proper mesopores by controlling $\mathrm{CO}_{2}$ activation time. As an electrode material, PIAC shows an excellent electrochemical performance with specific capacitance of up to $237 \mathrm{~F} / \mathrm{g}$ at current density of $100 \mathrm{~mA} / \mathrm{g}$ and capacitance retention of $86 \%$.
\end{abstract}

Key words: polyimide film; activated carbon; $\mathrm{CO}_{2}$ activation; high specific surface; electrochemical performance

收稿日期: 2017-01-22; 收到修改稿日期：2017-05-18

基金项目：国家自然科学基金(21436009, 21376037); 中央高校基本科研业务费(DUT14RC(3)049)

National Natural Science Foundation of China (21436009, 21376037); Fundamental Research Funds for the Central Universities (DUT14RC(3)049)

作者简介: 王 浩(1990-), 女, 硕士研究生. E-mail: wanghao9014@mail.dlut.edu.cn

通讯作者: 王同华，教授. E-mail: wangth@dlut.edu.cn 
聚酰亚胺(Polyimide, PI) 薄膜是一种新型的高 性能绝缘材料 ${ }^{[1]}$, 具有优良的电气性能、阻燃性能、 耐高温和耐辐射等特性, 被广泛应用于航空航天、电 子工业等领域, 成为最具前景的绝缘材料之一[2-3]。 然而, 随着 PI 薄膜市场需求量的日益增长, 在其应 用过程中产生大量的边角余料亟需处理。但 PI 薄膜 无法生物降解, 目前主要处理方法是碱降解、焚烧 和掩埋, 这既浪费资源, 又有可能对环境造成二次 污染。近年来, 我国环境污染严重, 低碳环保已经成 为我国发展的战略目标。因此, 开发新型的 PI 废弃 物处理技术, 实现其资源化利用是 PI 废弃物有效利 用的发展方向。

活性炭是一种多孔炭材料, 具有发达的孔隙结 构、高比表面积及丰富的表面功能基团, 通常作为 吸附剂、催化剂或催化剂载体等被广泛应用于工业 生产的各个领域, 特别是其良好的导电性和化学稳 定性, 一直是制造超级电容器和动力电池电极的首 选材料 ${ }^{[4-6]}$ 。PI 薄膜因含碳量高、灰分小、有丰富的 表面氧氮官能团等优点成为制备高性能活性炭的优 质前驱体材料, 所以利用 PI 薄膜制备高级活性炭是 实现 PI 薄膜资源化利用, 变废为宝的有效途径。

本工作以 PI 薄膜边角料为原料, 采用物理活化 法制备高比表面活性炭, 研究其吸附性能和结构特 性, 探讨活化工艺对孔结构特性的影响。在此基础 上, 进一步探讨 PI 基活性炭的电化学性能及孔结构 对其电化学性能的影响, 为其作为超级电容器电极 材料提供依据。

\section{1 实验方法}

\section{1 活性炭的制备}

在氮气气氛下, 将 PI 废膜放置于管式炭化炉中 以 $5^{\circ} \mathrm{C} / \mathrm{min}$ 升温至 $700^{\circ} \mathrm{C}$ 炭化 $1 \mathrm{~h}$, 炭化后的样品记 为 PIC。再对样品 PIC 进行 $\mathrm{CO}_{2}$ 活化, 活化温度为 $880^{\circ} \mathrm{C}$, 活化时间分别为 $2 、 4 、 6$ 和 $8 \mathrm{~h}$, 活化后的样 品记为 PIAC- $t$ ( $t$ 代表活化时间)。

\section{2 吸附性能测试}

按照 GB/T 12496.8-1999 测定活性炭的碘吸附 值, 苯吸附值采用静态吸附法进行测定, 具体测试 方法见文献[7]。

\section{3 结构表征}

采用美国 Quantachromecom 公司全自动物理吸 附仪(Autosorb iQ2)测试活性炭的孔结构, 之后分别 利用 BET 方程、HK 法、DFT 法、t-plot 方法计算 样品的比表面积 $\left(S_{\mathrm{BET}}\right)$ 、孔径分布以及微孔孔容 $\left(V_{\text {mic }}\right)$ 。当相对压力为 0.98 时, 样品所吸附液氮体积
对应其总孔容 $\left(V_{\mathrm{tot}}\right)$ 。采用日本 Rigaku 公司 $\mathrm{X}$ 射线 衍射仪(D/Max-2400 X) 分析活性炭的微结构。利用 美国 Thermo Fisher 公司 $\mathrm{X}$ 射线光电子能谱仪 (Escalab 250Xi)测试活性炭的表面化学结构。

\section{4 电化学性能测试}

利用三电极体系测试样品的电化学性能。将 PIAC、粘结剂(聚四氟乙烯悬浊液)、导电炭黑按质 量比为 $8: 1: 1$ 混合均匀, 压制成圆形电极片, 干燥 后将电极片压在两片泡沫镍中间作为工作电极, 铂 电极和 $\mathrm{Hg} / \mathrm{HgO}$ 电极分别作为对电极和参比电极, 电解液为 $6 \mathrm{~mol} / \mathrm{L}$ 的 $\mathrm{KOH}$ 溶液。测试前, 先将工作 电极在电解液中浸泡 $24 \mathrm{~h}$ 以确保电解质充分浸润在 电极材料中。采用上海辰华公司电化学工作站 $(\mathrm{CHI}$ 660D)测试样品的循环伏安 $(\mathrm{CV})$ 、恒流充放电(GCD) 和交流阻抗(EIS)。根据恒流充放电曲线计算质量比 电容 $C_{\mathrm{g}}(\mathrm{F} / \mathrm{g})$, 其公式如下:

$$
C_{g}=I \Delta t /(m \Delta V)
$$

其中, $I$ 为放电电流, $\mathrm{A} ; \Delta t$ 为放电时间, $\mathrm{s} ; m$ 为活性物 质的质量, $\mathrm{g} ; \Delta V$ 为放电时间内的压降, $\mathrm{V}$ 。

\section{2 结果与讨论}

\section{1 活化时间对 PIAC 吸附性能及孔结构影响}

\subsection{1 活化时间对吸附性能影响}

图 1 为 $\mathrm{CO}_{2}$ 活化时间对活性炭吸附性能的影响, 可以看出, 随着 $\mathrm{CO}_{2}$ 活化时间的延长, 由 $\mathrm{PI}$ 薄膜制 备的活性炭收率降低, 碘和苯的吸附值却大幅提 高。当活化时间为 $4 \mathrm{~h}$ 时, 碘和苯的吸附值就分别达 1500 和 $600 \mathrm{mg} / \mathrm{g}$, 表现出优异的吸附能力。一般来 说, 碘吸附值主要反映了孔径在 $1 \mathrm{~nm}$ 以上微孔的数 量, 而苯吸附值主要反映了孔径在 $0.6 \mathrm{~nm}$ 以上微孔 的数量 ${ }^{[8-9]}$ 。可见, PI 薄膜制备的活性炭具有丰富的 微孔结构。

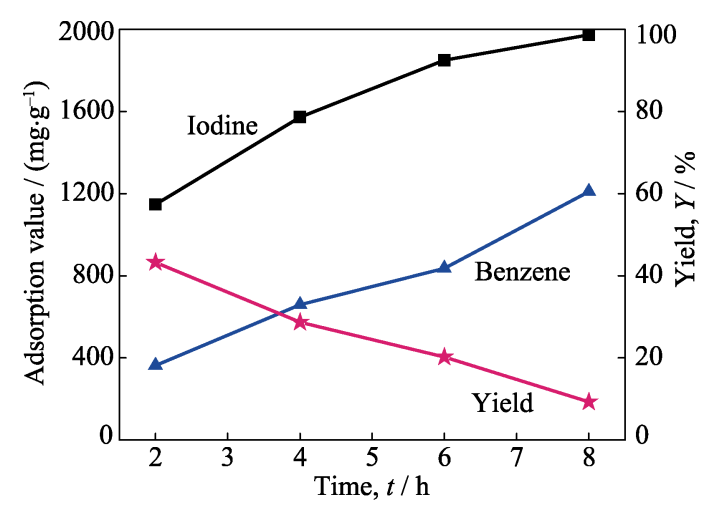

图 1 活化时间对 PIAC 吸附性能和产率的影响

Fig. 1 Effect of activation time on the adsorption properties and yield of PIAC 


\subsection{2 活化时间对孔结构影响}

利用 $\mathrm{N}_{2}$ 吸附探讨 $\mathrm{CO}_{2}$ 活化时间对 PIAC 孔结构 特性的影响, 图 2 给出了 PI 基活性炭的吸脱附等温 线和孔径分布, 它们的孔结构特性参数见表 1 。可以 看出, 随着 $\mathrm{CO}_{2}$ 活化时间的延长, PIAC 的吸脱附等 温线发生明显的变化。当活化时间小于 $4 \mathrm{~h}$ 时, 吸脱 附等温线为典型的 I 型等温线 ${ }^{[10]}$, 说明活性炭具有 丰富的微孔结构; 当活化时间超过 $4 \mathrm{~h}$ 后, 吸脱附等 温线呈现出 I 型和IV 型等温线的融合, 说明此时的 活性炭不仅微孔结构发达, 而且还具有介孔结构 (图 2(a))。由此可见, 延长活化时间, PIAC 的孔隙结 构得到不断丰富, 孔径变大, 孔径分布变宽(图 2(b)), 由最初的发达微孔结构转变为微一介孔结构。图 3 进一步给出了随着 $\mathrm{CO}_{2}$ 活化时间的延长, PIAC 孔径 尺度的变化规律, 可以看出, 活化初期所制备的活 性炭 PIAC-2 和 PIAC-4 均以微孔为主, 并且极微孔 (孔径小于 $0.7 \mathrm{~nm})^{[11]}$ 所占比重较大。随着活化时间 的延长, 活性炭的孔容积逐渐增大, 其中较大孔径 的孔容积明显增大, 但微孔容积并未明显的降低; 活化时间为 $6 \mathrm{~h}$ 时, 出现孔径大于 $2 \mathrm{~nm}$ 的介孔。继 续延长活化时间, 尺度在 $223 \mathrm{~nm}$ 的介孔所占比重 急剧增加, 此时的活性炭不仅具有发达的微孔结构, 而且介孔结构也比较丰富, 并且发现 PIAC 的孔隙 结构主要集中在 $0.5 \sim 1 \mathrm{~nm}$ 之间, 活化时间延长, 孔 径分布变宽。活化时间为 $8 \mathrm{~h}$ 所制备的活性炭比表 面积高达 $2809 \mathrm{~m}^{2} / \mathrm{g}$, 总孔容积达 $1.423 \mathrm{~cm}^{3} / \mathrm{g}$, 这充 分说明 PI 薄膜是制备高表面积活性炭的优质原料。

\subsection{3 活化机理分析}

物理活化法制备活性炭主要是利用活化剂与碳 发生氧化反应所生成的气体析出, 从而形成孔隙结 构。活化剂与碳的氧化反应是吸热反应, 其反应速 率主要取决于反应所需的热力学能量。

表 2 给出了不同前驱体经 $\mathrm{CO}_{2}$ 活化所制备的活 性炭的比表面积和孔容积, 可以发现, 以 $\mathrm{CO}_{2}$ 为活 化剂制备活性炭所需活化温度一般均在 $900^{\circ} \mathrm{C}$ 以上, 这是因为 $\mathrm{CO}_{2}$ 与碳的反应热力学能量较高 $(\Delta H=$ $171.5 \mathrm{~kJ} / \mathrm{mol}$ ), 只有活化温度较高时才能在相对较 短的时间内达到理想的活化效果, 制备出孔隙发达
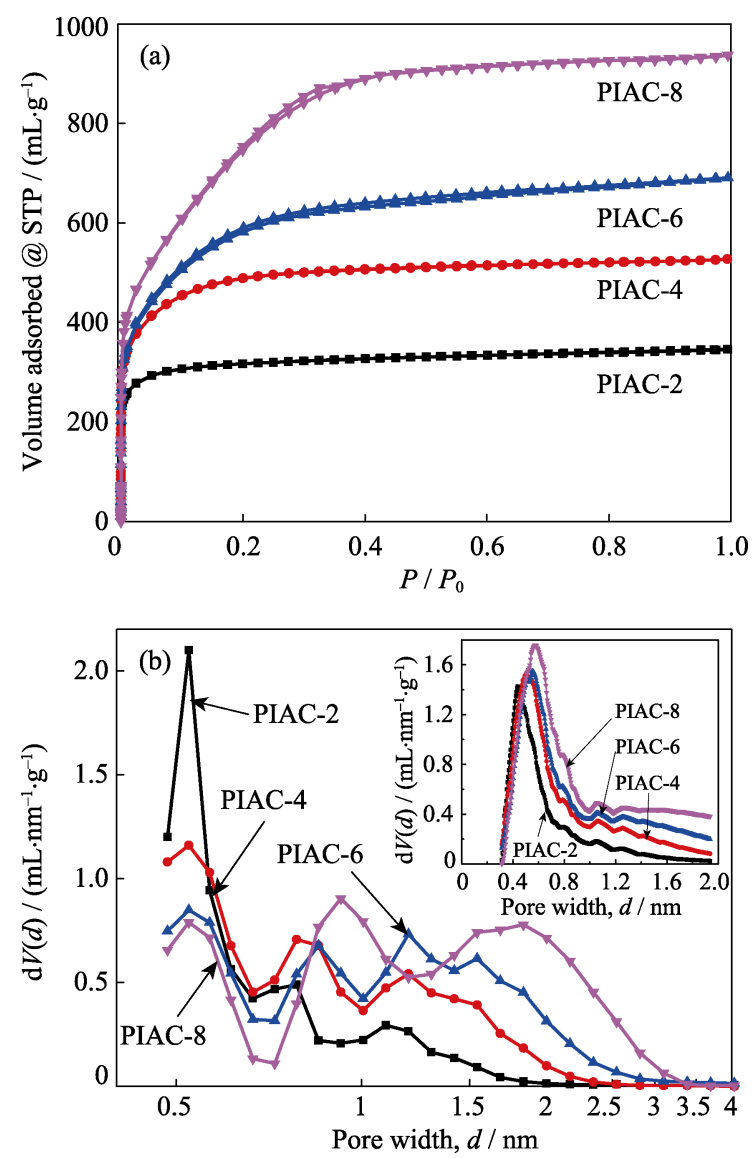

图 2 PIAC 的(a) 吸脱附等温线和(b) DFT 孔径分布曲线 Fig. $2 \mathrm{~N}_{2}$ adsorption / desorption isotherms (a) and pore size distributions (b) of DFT

Inset is the micropore distributions by HK equation for PIAC

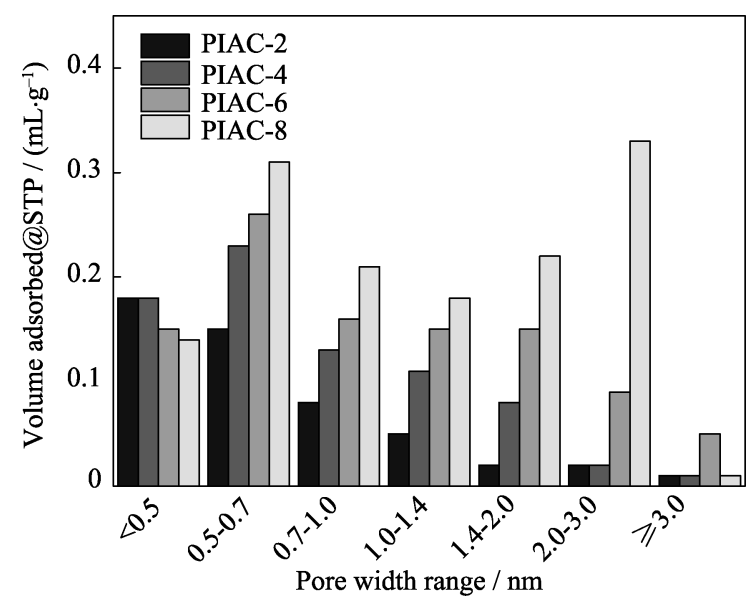

图 3 PIAC 的孔径分布柱状图

Fig. 3 Histogram of pore size distributions for PIAC

表 1 PIAC 的孔结构参数

Table 1 Porous textural parameters deduced from $\mathbf{N}_{2}$ adsorption for PIAC

\begin{tabular}{ccccccc}
\hline Sample & $S_{\mathrm{BET}} /\left(\mathrm{m}^{2} \cdot \mathrm{g}^{-1}\right)$ & $V_{\mathrm{tot}} /\left(\mathrm{cm}^{3} \cdot \mathrm{g}^{-1}\right)$ & $V_{\mathrm{mic}} /\left(\mathrm{cm}^{3} \cdot \mathrm{g}^{-1}\right)$ & $V_{\text {meso }} /\left(\mathrm{cm}^{3} \cdot \mathrm{g}^{-1}\right)$ & $\left(V_{\text {meso }} / V_{\text {tot }}\right) / \%$ & $C_{\mathrm{g}} /\left(\mathrm{F} \cdot \mathrm{g}^{-1}\right)$ \\
\hline PIAC-2 & 1229 & 0.510 & 0.484 & 0.026 & 5 & 182 \\
PIAC-4 & 1811 & 0.792 & 0.737 & 0.055 & 7 & 212 \\
PIAC-6 & 2120 & 1.043 & 0.854 & 0.189 & 25 & 237 \\
PIAC-8 & 2809 & 1.423 & 1.067 & 0.356 & 25 & 233 \\
\hline
\end{tabular}


表 2 采用不同前驱体的活性炭性能比较

Table 2 Comparison of performance of AC prepared from different precursors

\begin{tabular}{ccccc}
\hline Precursor & $T /{ }^{\circ} \mathrm{C}$ & Time $/ \mathrm{h}$ & $S_{\mathrm{BET}} /\left(\mathrm{m}^{2} \cdot \mathrm{g}^{-1}\right)$ & $V_{\text {tot }} /\left(\mathrm{cm}^{3} \cdot \mathrm{g}^{-1}\right)$ \\
\hline Coconut shells $^{[12]}$ & 900 & 5.0 & 1653.0 & 1.045 \\
Anthracite $^{[13]}$ & 920 & 3.0 & 1071.0 & 0.45 \\
Depleted fullerene soot $^{[14]}$ & 900 & 2.0 & 938.0 & 0.56 \\
PFA $^{[15]}$ & 900 & 5.0 & 1150.0 & 0.45 \\
Corn stalk acid hydrolysis residue $^{[16]}$ & 1000 & 3.5 & 845.4 & - \\
Coffee endocarp & & 1038.0 & 0.460 \\
PI film in this work & 900 & 2.0 & 1229.0 & 0.510 \\
PI film in this work & 880 & 4.0 & 1811.0 & 0.792 \\
\hline
\end{tabular}

的活性炭。然而, 以 PI 薄膜为原料制备活性炭, 当 活化温度为 $880^{\circ} \mathrm{C}$ 时, 就可以制备出具有较高比表 面积的活性炭, 而且活性炭具有发达的微孔结构和 丰富的介孔。这是由于 PIC 是二维结构的薄膜, 厚 度在 $100 \mu \mathrm{m}$ 以下, 在活化过程中氧化气体 $\mathrm{CO}_{2}$ 由气 相主体向膜表面及所形成的孔内表面的扩散传递路 径明显缩短, 有利于更多 $\mathrm{CO}_{2}$ 分子在反应表面的吸 附、反应和扩散，加快反应速率，降低反应所需的热 力学能量, 从而可在较低的活化温度下制备出具有 丰富孔结构、高比表面和高孔容的活性炭。这也充 分说明了 PI 薄膜是制备高比表面活性炭的优质前 驱体材料。

基于 PI 薄膜的二维结构更利于活化气体分子 的接触, 从而达到充分活化, 实现比表面积和孔容 积的大幅增大的特性。本课题组认为基于 PI 薄膜制 备活性炭的活化机理主要分为两个阶段：(1)极微孔 的形成，首先活化气体分子在薄膜表面吸附与碳原 子发生活化反应，刻蚀形成极微孔; (2)极微孔的扩 张，随着活化时间的延长，活化气体分子在薄膜表 面不断反应刻蚀形成更多极微孔的同时，还会有大 量的活化气体分子进入形成的微孔内部，在孔壁上 发生活化反应，使极微孔的孔道不断扩张形成更大 的微孔, 并进一步形成介孔。在活化过程, 两个阶段 同时进行，不断有极微孔形成，同时还有极微孔逐 渐扩宽形成较大的微孔和介孔。这就是为什么 PI 基活性炭不仅具有发达的极微孔结构，而且还具有 丰富的介孔结构和较高的比表面积和孔容积的原 因。

\section{2 活化时间对 PIAC 微结构影响}

图 4 给出了 PIAC 的 XRD 图谱。PIAC 有相似 的 XRD 图谱, 在 $2 \theta=24.5^{\circ}$ 处有明显的衍射峰, 对应 石墨炭微晶(002)晶面的特征衍射峰, 而在 $2 \theta=44^{\circ}$ 归 属于(100)晶面的衍射峰不明显, 表明所制备的 PI 基活性炭具有典型的炭无定型微晶质结构特征 ${ }^{[18]}$,
其微晶化程度较弱 ${ }^{[14]}$ 。随着 $\mathrm{CO}_{2}$ 活化时间的延长, (002) 衍射峰强度逐渐减弱, 表明 $\mathrm{CO}_{2}$ 对 PI 基活性炭 的刻蚀程度增加 ${ }^{[19]}$, 微晶的数目减少。但通过 XRD 数据计算得到的层间距 $\left(d_{002}\right)$ 数值相当, 表明活化时 间对 $d_{002}$ 影响较小，这与 Farma 等 ${ }^{[6]}$ 所得结论一致。

\section{3 活化时间对表面化学结构的影响}

采用 $\mathrm{X}$ 射线光电子能谱(XPS)测试确定样品的 表面化学结构, 如图 5 所示。从图 5(a)可以看出, N1s 谱图可以去卷积分为三个不同类型的特征峰, 分别 代表三种不同类型的含氮官能团，并标记为六元环 的吡啶类氮 $(\mathrm{N}-6,398.4 \mathrm{eV})$; 四元氮 $(\mathrm{N}-\mathrm{Q},(400.9 \pm$ $0.5) \mathrm{eV}$ )和吡啶氮官能团 $(\mathrm{N}-\mathrm{X}, 402 \sim 405 \mathrm{eV})$ 。前驱体 PIC 中含有三种类型的含氮官能团, 这是前驱体特 有的官能团结构。随着活化的进行, 总含氮量从 $3.7 \mathrm{wt} \%$ 减少至 $1.7 \mathrm{wt} \%$, 这主要是由于脱氮作用和芳 构化造成的 ${ }^{[20]}$ 。随着 $\mathrm{CO}_{2}$ 活化时间的延长, 其化学 键合发生变化, 活化后的样品 N-6、N-X 含量增加, 而 N-Q 含量减少，表明随着活化时间的延长，N-Q 逐渐向 N-X 和 N-6 形式转换。根据图 5(b)PIAC 的 O1s 的 XPS 去卷积扫描谱图, 可以确定样品表面的 含氧官能团。其中氧原子主要存在形式分别标记为: $\mathrm{O}-\mathrm{I}(\mathrm{C}=\mathrm{O}$ 和/或醌基氧, $(530.8 \pm 0.3) \mathrm{eV})$; O-II $(\mathrm{C}-\mathrm{OH}$

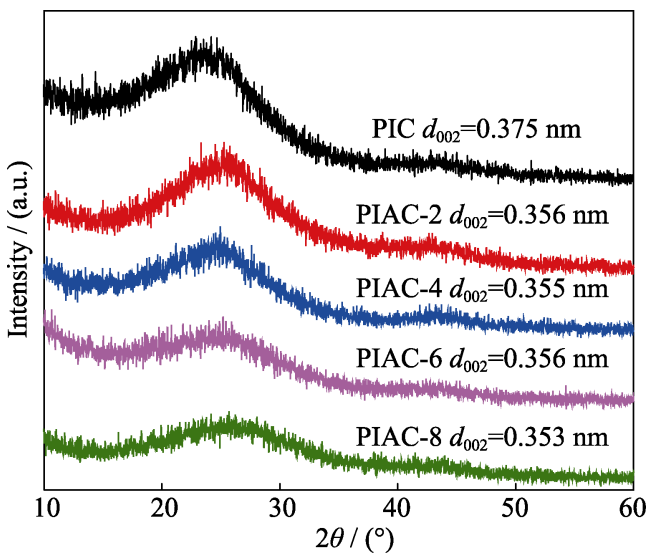

图 4 PIAC 的 XRD 图谱

Fig. 4 XRD patterns of PIAC 

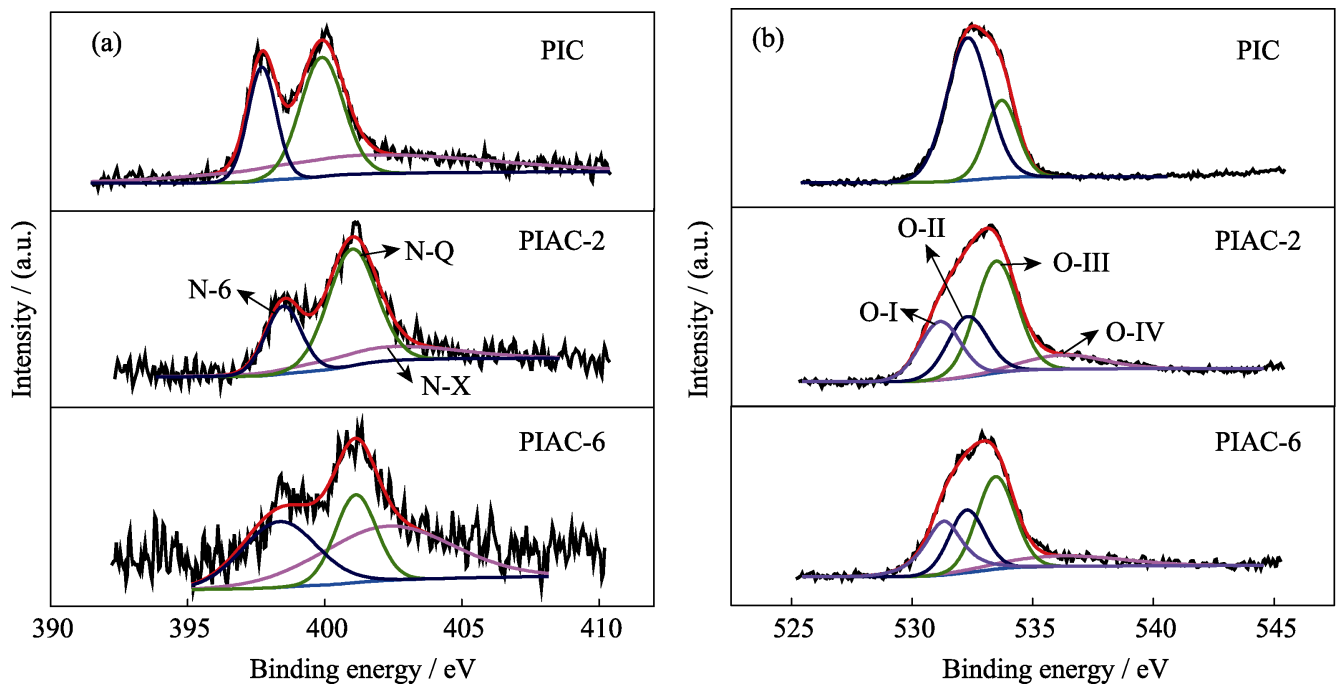

图 5 PIAC-t 的(a) N1s 谱图和(b) O1s 图谱

Fig. 5 N1s spectra (a) and O1s spectra (b) of PIAC- $t$

和/或 $\mathrm{C}-\mathrm{O}-\mathrm{C},(532.0 \pm 0.5) \mathrm{eV}) ; \mathrm{O}-\mathrm{III}(\mathrm{COO}-, 533.9$ $\pm 0.5 \mathrm{eV}$ ); $\mathrm{O}-\mathrm{IV}$ (化学吸附 $\mathrm{H}_{2} \mathrm{O} / \mathrm{O}_{2}$ 中的氧, $537 \mathrm{eV}$ )。 O-I 和 O-II 是有利于提高噟电容的氧化还原活性的 含氧官能团 ${ }^{[21]}$ 。通过 PIC 的 $\mathrm{O} 1 \mathrm{~s}$ 的谱图可以看出, 其 含氧官能团主要为 O-II 和 O-III, 这是 PI 薄膜的结构 特征峰。随着 $\mathrm{CO}_{2}$ 活化的进行，总含氧量从 $5.7 \mathrm{wt} \%$ 逐渐增加至 $7.1 \mathrm{wt} \%$, 这一方面可能是由于物理活化 引入了氧, 另一方面可能是因为高温活化, 造成 $\mathrm{PIC}$ 结构的重排。从图 5(b) 可以看出 $\mathrm{O} 1 \mathrm{~s}$ 的化学键 合发生变化, O-II 含量逐渐减少, 出现 O-I 和 O-IV 官 能团, 其中 $\mathrm{O}-\mathrm{I}$ 的形成是由于部分 $\mathrm{C}-\mathrm{O}-\mathrm{C}$ 断裂。

\subsection{PIAC 孔结构对电化学性能影响}

\subsubsection{PIAC 的电化学性能}

采用 $\mathrm{CV}$ 和 $\mathrm{GCD}$ 研究了 PI 基活性炭的电化学 性能, 结果如图 6.7 所示。图 6(a)为活性炭在 $2 \mathrm{mV} / \mathrm{s}$ 扫速下的循环伏安曲线, 可以看出, 所制备活性炭 电极的 CV 曲线均呈现无明显氧化还原峰的类矩形,
说明电荷主要存储于电极/电解液界面形成的双电 层中 ${ }^{[19]}$ 。延长活化时间, CV 曲线的面积先增加后减 小, 表明比电容值先增加后减小, 其中 PIAC-6 有最 大的比电容。测试 PIAC-6 在不同扫速下的循环伏 安特性, 结果如图 6(b)所示。当扫速从 $2 \mathrm{mV} / \mathrm{s}$ 增加 至 $300 \mathrm{mV} / \mathrm{s}$ 时, $\mathrm{CV}$ 曲线的形状没有明显的变化, 说 明电荷的储存主要依赖于电极和电解液界面所形成 的双电层, 电极具有良好的快速充放电特性。由图 7(a)可以看出, PI 基活性炭在 $100 \mathrm{~mA} / \mathrm{g}$ 的电流密度 下的恒流充放电曲线均有相似的形状，即由接近相 等的充放电时间形成的对称性。这种对称性表明 PI 基活性炭具有较高的亲水性, 与电解液接触良好 ${ }^{[6]}$ 。 同时可以看出, PIAC 具有较小的 $\Delta I R$, 说明其有微 弱的噟电容, 这部分赝电容主要来源于热稳定的羰 基、醌基和吡啶型含氧官能团 ${ }^{[22-23]}$ 。对比不同 PI 基活性炭的 GCD 曲线发现，其充放电时间各不相 同，其中 PIAC-6 的充放电时间最长，表明它具有最
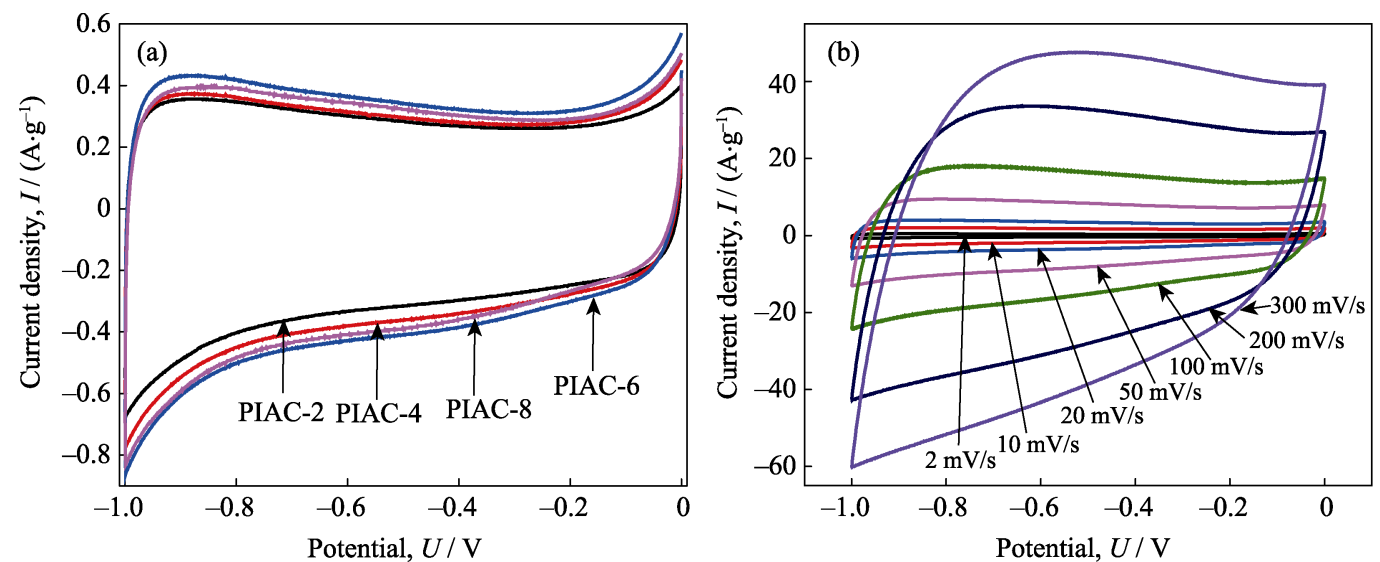

图 6 (a) PIAC- $t$ 在 $2 \mathrm{mV} / \mathrm{s}$ 下的循环伏安曲线和(b)PIAC- 6 在不同扫速下的循环伏安曲线

Fig. $6 \mathrm{CV}$ curves of PIAC- $t$ at scan rate of $2 \mathrm{mV} / \mathrm{s}$ (a) and PIAC-6 at different scanning rates (b) 

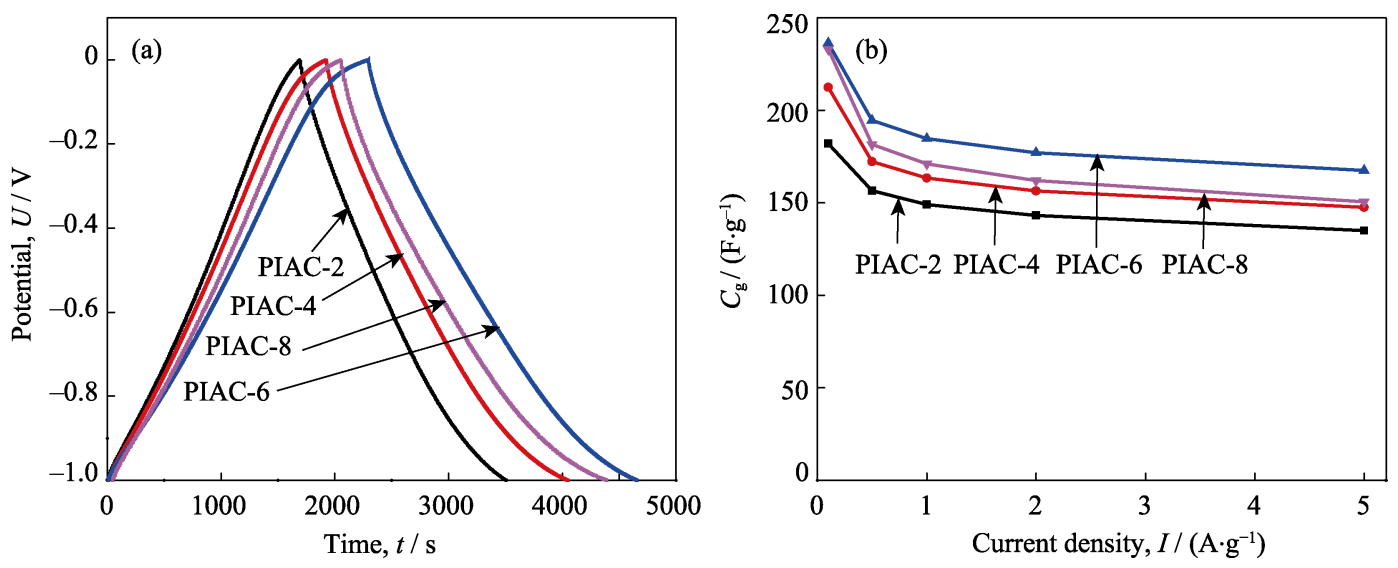

图 7 PIAC 在(a) $100 \mathrm{~mA} / \mathrm{g}$ 电流密度下的恒流充放电和(b)不同电流密度下的质量比电容

Fig. 7 GCD of PIAC at current density of $100 \mathrm{~mA} / \mathrm{g}$ (a) and specific capacitance of PIAC at different current densities (b)

大的比电容值, 这和 $\mathrm{CV}$ 结果一致。通过公式(1)计 算得到的 PIAC-2、PIAC-4、PIAC-6 和 PIAC-8 在不 同电流密度下的质量比电容如图 7(b)所示, 可以看 出, 当电流密度从 $100 \mathrm{~mA} / \mathrm{g}$ 增加至 $5 \mathrm{~A} / \mathrm{g}$ 时, 比电 容值有所下降。以 $0.5 \mathrm{~A} / \mathrm{g}$ 为基准计算得到的四个样 品的比电容值分别为 $86 \% 、 86 \% 、 86 \%$ 和 $83 \%$ 。

如图 8(a)所示为 PIAC 在 $6 \mathrm{~mol} / \mathrm{L} \mathrm{KOH}$ 中的 Nyquist 谱图, 插图为高频区放大图。该图由高频 区、中频区及低频区三部分组成。高频区半圆弧的 存在是多孔材料的典型电容特征, 代表主要的电阻 影响和在电极/电解液界面上微弱的电容影响 ${ }^{[6]}$ 。随 着活化时间的延长, 半圆弧的半径逐渐增大, 表明 电极的阻抗增加。PIAC 在低频区有相近的较大的斜 率, 说明电解液离子在电极材料微孔中的扩散阻力 随着频率减小而减小, 从而证明样品均具有很好的 电容特性。图 8(b)为 PIAC 的 Bode 谱图, 其中 $45^{\circ}$ 相角对应的频率反映了电极的离子响应频率, 在此 特征频率下电容器的电阻和电容达到平衡。由图 8(b) 可看出, PIAC-2、PIAC-4、PIAC-6 和 PIAC- 8 的响
应频率分别为 $0.50 、 0.72 、 0.72$ 及 $0.39 \mathrm{~Hz}$ 。由于电 极的响应频率越大，响应速度越快，因此 PIAC-4 和 PIAC-6 具有最快的离子响应速度，表明在大电流下， 其表面利用率和电容保持率均较高。

\subsubsection{PIAC 结构特性对电化学性能影响}

研究表明, PIAC 的结构特性对其比电容、电容 保持率及阻抗均有影响, 主要影响因素为孔结构和 表面化学结构。

PIAC 孔结构对电化学性能的影响主要有比表 面积和孔结构。通常认为比表面积越大, 比电容值 越大，但两者并非线性关系; 孔径分布对比电容有 更直接的影响 ${ }^{[24-25]}$ 。研究表明孔径大于电解液离子 直径的孔对形成双电层有利 ${ }^{[26]}$ 。由于 $\mathrm{K}^{+}$和 $\mathrm{OH}^{-}$的水 合离子半径分别为 0.331 和 $0.3 \mathrm{~nm}$, 因此 $0.7 \mathrm{~nm}$ 以 上的孔有利于形成双电层。四个活性炭电极中大于 $0.7 \mathrm{~nm}$ 的孔容随活化时间的延长而增大, 因此其比 电容值逐渐增加。但 PIAC-8 出现反常, 这主要是由 于 PIAC-8 介孔所占比例远大于其他 PI 基活性炭, 造成孔容积浪费严重, 因此不利于高比电容的形
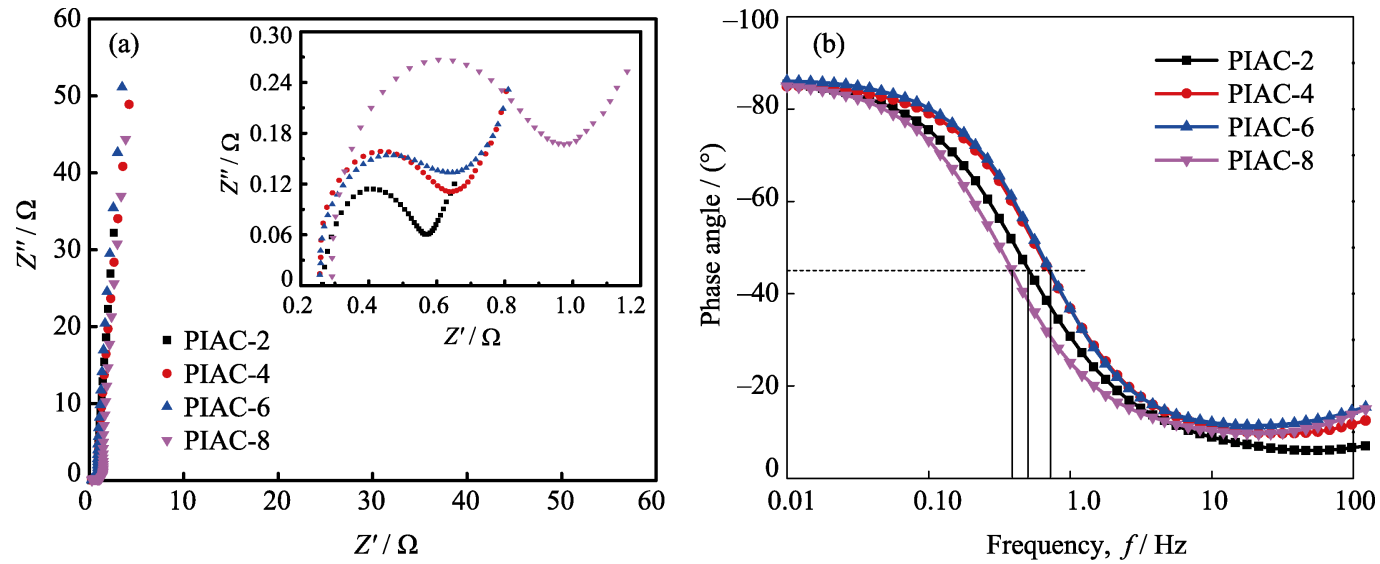

图 8 PIAC 的交流阻抗谱图

Fig. 8 AC impedance spectra of PIAC

(a) Nyquist plot with the inset showing the expanded high-frequency region; (b) Bode plot 
成。同时中孔率较高时, 不利于大电流充放电, 因此 PIAC-8 的电容保持率略低于其他 PI 基活性炭。

另外, 当样品微孔所占比重较大时, 其阻抗较 小, 响应速度快, 更利于大电流下表面的利用。因此, 当活性炭孔径集中于 $0.7 \sim 2 \mathrm{~nm}$, 并有适量的介孔时 有利于提高 PI 基活性炭在 $\mathrm{KOH}$ 电解液中的电化学 性能。

PIAC 表面化学结构的影响主要体现在两方面: (1) PIAC 表面含氧官能团有利于提高电极材料的亲 水性, 加强其与 $\mathrm{KOH}$ 电解液的接触, 增加形成双电 层的有效比表面积; (2) 发生在 PIAC 表面与边缘炭 相连的氮( $\mathrm{N}-\mathrm{X}$ 和 N-6)上的赝电容, 与由双电层结构 产生的电容相作用，从而提高电极材料的整体电容。

因此, PI 基活性炭良好的电化学性能是其孔结 构和表面化学结构特性协同作用的结果。

\section{3 结论}

以聚酰亚胺薄膜为炭前驱体, 采用 $\mathrm{CO}_{2}$ 物理活 化的方式成功制备了具有无定型微晶结构、孔隙结 构发达的 PI 基高比表面活性炭。其比表面积最高可 达 $2809 \mathrm{~m}^{2} / \mathrm{g}$, 总孔容积达 $1.423 \mathrm{~cm}^{3} / \mathrm{g}$ 。通过控制 $\mathrm{CO}_{2}$ 的活化时间可以有效调控活性炭的孔结构和孔 道尺度。作为超级电容器电极材料, PI 基活性炭显 示出良好的电化学性能, 其比电容在 $100 \mathrm{~mA} / \mathrm{g}$ 条件 下高达 $237 \mathrm{~F} / \mathrm{g}$, 以 $0.5 \mathrm{~A} / \mathrm{g}$ 计算得到的电容保持率 为 $86 \%$ 。并发现 PI 基活性炭的孔结构对其电化学性 能有一定的影响。当孔径集中于 $0.7 \sim 2 \mathrm{~nm}$, 并存在 适量的介孔时, 有利于提高样品在 $\mathrm{KOH}$ 电解液中 的电化学性能。利用 PI 薄膜废弃的边角余料制备高 表面活性炭不仅能够变废为宝, 而且能够提供一种 具有应用潜力的电极材料。

\section{参考文献:}

[1] MA C, SHI J L, LI Y J, et al. Preparation of nitrogen-enriched porous carbon nanofibers and their electrochemical performance as electrode materials of supercapacitors. New Carbon Materials, 2015, 30(4): 295-301.

[2] QIN Z X. The introduction of polyimide. Information Recording Materials, 2010, 11(5): 63-64.

[3] WU G G. The manufacture and application of polyimide and film. Information Recording Materials, 2010, 11(5): 47-53.

[4] LI B, DAI F, XIAO Q, et al. Nitrogen-doped activated carbon for a high energy hybrid supercapacitor. Energy \& Environmental Science, 2016, 9(1): 102-106.

[5] TAER E, DERAMAN M, TALIB I A, et al. Physical, electrochemical and supercapacitive properties of activated carbon pellets from pre-carbonized rubber wood sawdust by $\mathrm{CO}_{2}$ activation. Current Applied Physics, 2010, 10(4): 1071-1075.

[6] FARMA R, DERAMAN M, AWITDRUS A, et al. Preparation of highly porous binderless activated carbon electrodes from fibres of oil palm empty fruit bunches for application in supercapacitors. Bioresource Technology, 2013, 132(3): 254-261.

[7] WANG T H, JIAO T T, CHAI C L. A study of recovering gasoline vapor on activated carbon. Petroleum Processing and Petrochemicals, 2009, 40(9): 60-65.

[8] SIRCAR S, GOLDEN T C, RAO M.B. Activated carbon for gas separation and storage. Carbon, 1996, 34(1): 1-12.

[9] YUE Z R, JIANG W, WANG L, et al. Surface characterization of electrochemically oxidized carbon fibers. Carbon, 1999, 37(11): $1785-1796$.

[10] SI W J, WU X Z, XING W, et al. Bagasse-based nanoporous carbon for supercapacitor application. Journal of Inorganic Materials, 2011, 26(1): 107-112.

[11] HU C, SEDGHI S, MADANI S H, et al. Control of the pore size distribution and its spatial homogeneity in particulate activated carbon. Carbon, 2014, 78(18): 113-120.

[12] YANG K B, PENG J H, XIA H Y, et al. Preparation of coconut shells-based activated carbons with $\mathrm{CO}_{2}$ activation. Carbon Techniques, 2010, 29(1): 20-23.

[13] 袁翠翠. $\mathrm{CO}_{2}$ 活化制备煤基微孔活性炭的研究. 徐州: 中国矿业. 大学硕士学位论文, 2016.

[14] SUN L, WANG C L, ZHOU Y, et al. Activated carbons produced form depleted fullerene soot by carbon dioxide activation and their electrochemical properties. New Carbon Materials, 2014, 29(2): 55-60.

[15] QAJAR A, MARYAM P, RAJAGOPALAN R, et al. Surface compression of light adsorbates inside microporous PFA- derived carbons. Carbon, 2013, 60(12): 538-561.

[16] SI H Y, SUN K, CHEN L, et al. Orthogonal experimental study on activated carbon from biomass hydrolysis residues activated by carbon dioxide. Chemical Industry and Engineering Progress, 2014, 33(s1): 141-144.

[17] NABAIS J M V, TEIXEIRA J G, ALMEIDA I. Development of easy made low cost bindless monolithic electrodes from biomass with controlled properties to be used as electrochemical capacitors. Bioresource Technology, 2011, 102(3): 2781-2787.

[18] YANG H, GONG M C, CHEN Y Q. Effect of size distribution on adsorption capacities of activated carbons for $\mathrm{CH}_{4}$ and $\mathrm{CO}_{2}$. Chinese Journal of inorganic Chemistry, 2011, 27(6): 1053-1058.

[19] DENG M G, WANG R Q, FENG Y H. Effect of petroleum coke expanding by $\mathrm{HNO}_{3}$ on the performance of supercapacitor based on the activated carbon. Journal of Inorganic Materials, 2014, 29(3): 245-249.

[20] FENG Y Q, TANG F L, LANG J W, et al. Facile approach to preparation of nitrogen-doped graphene and its supercapacitive performance. Journal of Inorganic Materials, 2013, 28(6): 677-682.

[21] DENISA H J, MYKOLA S, GAO Q L, et al. Combined effect of nitrogen- and oxygen-containing functional group of microporous activated carbon on its electrochemical performance in supercapacitors. Advanced Functional Materials, 2009, 19(3): 438-447.

[22] MONTES-MORÁN M A, SUÁREZ D, MENÉNDEZ J A, et al. On the nature of basic sites on carbon surfaces: an overview. Carbon, 2004, 42(7): 1219-1225.

[23] HUANG W, ZHANG Y, BAO S, et al. Desalination by capacitive deionization process using nitric acid-modified activated carbon as the electrodes. Desalination, 2014, 340(1): 67-72.

[24] VIX-GUTERL C, FRACKOWIAK E, JUREWICZ K, et al. Electrochemical energy storage in ordered porous carbon materials. Carbon, 2005, 43(6): 1293-1302.

[25] GRYGLEWICZ G, MACHNIKOWSKI J, LORENC G E, et al. Effect of pore size distribution of coal-based activated carbons on double layer capacitance. Electrochimica Acta, 2005, 50(5): 11971206.

[26] LAUŠEVIĆ Z, APEL P Y, KRSTIĆ J B, et al. Porous carbon thin films for electrochemical capacitors. Carbon, 2013, 64: 456-463. 